\title{
Genetic parameters of in-vivo prediction of carcass, head and fillet yields by internal ultrasound and 2D external imagery in large rainbow trout (Oncorhynchus mykiss) is
}

\author{
Pierrick Haffray ${ }^{a, *}$, Jérome Bugeon ${ }^{b}$, Quentin Rivard ${ }^{a}$, Benjamin Quittet $^{a}$, Sophie Puyo ${ }^{a}$, \\ Jean Michel Allamelou ${ }^{\mathrm{c}}$, Marc Vandeputte ${ }^{\mathrm{d}, \mathrm{e}}$, Mathilde Dupont-Nivet ${ }^{\mathrm{d}}$
}

\author{
a SYSAAF, LPGP/INRA, Campus de Beaulieu, 35042 Rennes, France \\ b INRA, UR1037, LPGP, SFR Biosit, Campus de Beaulieu, F-35000 Rennes, France \\ ${ }^{c}$ LABOGENA, Domaine de Vilvert, F-78350 Jouy-en-Josas, France \\ d INRA, UMR1313 Génétique Animale et Biologie Intégrative, F-78350 Jouy-en-Josas, France \\ ${ }^{\mathrm{e}}$ Ifremer, UMR110 INTREPID, F-34250 Palavas-les-Flots, France
}

*: Corresponding author : Pierrick Haffray, tel.: + 33223485378 ; fax: + 33223485020 ;

email address : haffray@rennes.inra.fr

\begin{abstract}
:
Selection to improve processing yields relies on sib selection, in which live candidates are ranked according to their family breeding value. This approach limits genetic progress, as it only exploits genetic variability between families and not within them. Indirect criteria measured on live candidates could overcome this limitation. The present study (1) proposes a procedure to identify indirect criteria to predict processing yields in rainbow trout (head, carcass and fillet yields), (2) estimates genetic parameters of these indirect criteria, and (3) predicts relative genetic gains in processing yields using full-sib selection or indirect individual selection on those indirect criteria.
\end{abstract}

DNA-pedigreed all-female rainbow trout Oncorhynchus mykiss $(n=2029,1631.0 \pm 355.6 \mathrm{~g})$ from 600 families produced from 100 sires and 60 dams were characterized by external and internal non-lethal morphological measures using digital pictures and real time ultrasound tomography. Nineteen landmarks were recorded on the digital pictures to define the outline of the body, head and lateral line. Their coordinates were used to calculate different lengths, heights and areas. Five different internal thicknesses were measured by ultrasound tomography.

In the first phase of this study, processing yields were predicted using multiple linear regressions including both external and internal morphometric variables. In a second phase, the heritability of the predicted values and their genetic correlations with real processing yields were estimated using animal models. Predicted yields exhibited intermediate heritabilities $(0.25-0.28)$ that were half the value of heritabilities for real processing yields $(0.47-0.55)$, but had high genetic correlations with these real yields $(0.87-0.90)$. The relative efficiency of indirect selection (IS) on these indirect criteria was compared to theoretical mass selection (MS) or sib selection (FS) with different family sizes (10 or $100)$ and two different selection pressures $(10 \%$ or $40 \%)$. At the same selection pressure $(10 \%$, with 100 sibs per family \%), full-sib selection created genetic progress $49.6 \%$ to $60.5 \%$ higher than indirect selection according to the processing yield targeted. However, when sib-selection pressure was limited to a more realistic between family selection pressure $(40 \%$ and 10 sibs per family), indirect selection with $10 \%$ selection pressure was $21.9 \%$ to $32.7 \%$ more efficient than sib selection. 


\section{Highlights}

Application of ultrasound and 2D imagery to predict processing yields in rainbow trout. Estimation of genetic parameters and genetic correlations with yields to predict. Comparison of different strategy of selection.

Keywords: Salmonids ; Heritability ; Fillet yield ; Selection ; Aquaculture ; Morphometry

is Paper presented at the International Symposium on Genetics in Aquaculture XI, Auburn, AL, USA, June 24-30, 2012. 


\section{Introduction}

During the last twenty years (1987-2007), the proportion of rainbow trout Oncorhynchus mykiss slaughtered at a higher body weight than $300 \mathrm{~g}$ increased from $9 \%$ to $65 \%$ in France (Agreste, 2009). This increase in slaughtering weight up to $1-3.5 \mathrm{~kg}$ was driven by the consumer's demand for fresh or smoked fillet. It was allowed by important technological investments such as water oxygenation, use of extruded feed, vaccination, all-female monosexing, sterilization by triploid induction and selective breeding. Initially validated experimentally in brown trout Salmo trutta to improve growth (Chevassus et al., 2004), the optimized PROSPER procedure of mass selection was transferred to the farmers of rainbow trout starting in 1993 (Haffray et al., 2004). In 1996, gutted carcass yield was included in the selected traits based on individual scoring of fish morphology for more "salmon like" and elongated body shape. DNA parentage assignment with microsatellite markers was introduced in 1998 to optimize mating and to manage inbreeding. Since 2004, DNA fingerprinting was used to initiate sib selection on carcass yield based on intermediate heritability estimated with families mixed together since eyed stage and a posteriori DNApedigreed (Haffray et al., 2012b).

In the absence of high density SNP information that would allow within-family genomic selection, selection to improve processing yields is for the time being limited in practice to sib-selection to rank live candidates on their family breeding value estimated on slaughtered sibs. However, such a selection limits genetic progress as it uses only between-sire and between-dams additive variation and not within-family variation. The development of indirect criteria measured on live candidates could overcome this limit through the use of withinfamily variation to increase genetic gains through indirect selection with higher selection intensity than sib-selection. However, the relative advantage of sib- or indirect- selection depends on the genetic parameters of the traits but also on the number of sibs measured (Falconer and Mackay, 1996).

Across fish species, some authors have estimated heritability of single or combined external or internal body shape traits and their genetic correlations with weights or yields of carcass or fillets (Gjerde and Shaeffer, 1989; Rye and Refstie, 1995; Neira et al., 2004; Rutten et al., 2005; Kocour et al., 2007). However, heritabilities for these criterias and their genetic correlations with the yields to predict were in general limited. Moreover, internal measures were done on slaughtered sib and could not be used on live candidates. Ultrasound imagery presents a high potential for the in-vivo internal morphometric measures and prediction of processing yields in fish (Bosworth et al., 2001) but, as far as we know, there is no publication reporting its application to fish breeding to improve processing yields.

The present publication reports 1) a procedure to identify external and internal traits (measured by ultrasounds) to be combined by linear regression in indirect criteria to predict processing yields (head, carcass or fillet yields) 2) heritability of these criteria and their genetic correlation with the yield to predict. 3) predicted genetic progress according to sib or indirect selection on theses indirect criteria. This study used large all-female rainbow trout reared in fresh water in commercial French conditions of production. The results are expected to be useful to increase the efficiency of selection on processing yields. 


\section{Material and methods}

\subsection{Production of experimental fish and DNA collection}

The fish are the same as those reported in Haffray et al. (2012a, b). Briefly, they were derived from a commercial line from the Aqualande breeding company (France), already mass selected for growth for three generations using PROSPER procedure principles (Chevassus et al., 2004) and on external morphology (individual visual scoring). Pedigree was known with DNA parentage assignment. Inbreeding $(F)$ level estimated during three generations with the "pedigree" package of the R software was $0.45 \%$.

Briefly and as reported previously (Haffray et al., 2012a and b), 600 full-sib families were produced at the Aqualande breeding centre (Pissos, France). Sixty dams (two years old) were crossed with 100 sex-reversed females (defined as sires) in 10 full factorial crosses of six dams by 10 sires following the recommendations of Dupont-Nivet et al. (2006). At the eyed stage, 12 groups of five spawns each were created based on homogeneity for mean eyed egg size. The initial differences of mean weight between groups were progressively decreased by using different feeding ratios for each group (Haffray et al., 2012a). When groups achieved the same mean body length (147 days post fertilization, dpf), 250 individuals per group were pooled (in total 3000 fish). At $198 \mathrm{dpf}$, fish were individually tagged with RFID transponders. Their DNA was collected through a fin sample preserved in 95\% ethanol. At $220 \mathrm{dpf}$, fish were transferred to the "Viviers de la Hountine" fish farm (BelinBéliet, France) located $12 \mathrm{~km}$ downstream the Pissos hatchery. Fish were reared in fibreglass tanks until they reached $0.5 \mathrm{~g}$ and then in concrete raceways until the end of the experiment. The water temperature varies from 3 to $20^{\circ} \mathrm{C}$ and oxygen concentration was not limiting ( $>80 \%$ saturation). During growth, fish were fed to satiation using extruded commercial feed (Le Gouessant, Lamballe, France). Density increased progressively to reach $70 \mathrm{~kg} / \mathrm{m}^{3}$. Survival rate was $92.6 \%$ from tagging (d198) to slaughtering (d509).

\subsection{Processing trait recording}

The slaughtering protocol detailed in Haffray et al. (2012b) limited the post-mortem storage duration to less than five days. Fish $(n=2042)$ were randomly slaughtered at 509 or $511 \mathrm{dpf}$. The two sub-groups were treated in the same way: three days of fasting, live transportation by truck to the Aqualande processing plant (Roquefort, France, $50 \mathrm{~km}$ from the fish farms) for slaughter $\left(\mathrm{CO}_{2}\right.$ anaesthesia, followed by bleeding in icy water), and then transportation by refrigerated truck to IFREMER (Nantes, France) for processing and data collection. Data collection at fish processing was done between two to four days after slaughter. Malformed fish $(n=18)$ evaluated by visual examination of the vertebral axis after filleting were excluded from the measurements. Four other fish were discarded due to errors in data collection.

Non malformed fish ( $n=2020)$ were assigned to their parents by the French laboratory for livestock genotyping LABOGENA (ISO 17025 accredited, Jouy-en-Josas, France) using microsatellites markers. The rate of correct assignment to one unique parental pair with a maximum of one mismatch authorized was $99.5 \%$ and 559 full-sib families were represented out of the 600 expected (Haffray et al., 2012 b). All sires and all dams were represented. Spontaneous triploids $(n=48)$, identified from their DNA-fingerprint signature, were removed from the statistical analysis (see Haffray et al., 2012a).

Body length (BL), body weight (BW), Fulton coefficient $\mathrm{K}\left(=\mathrm{BW}(\mathrm{g})\right.$ * $\left.100 / \mathrm{BL}^{3}(\mathrm{~cm})\right)$, head yield $(\mathrm{Head} \%)$, gutted carcass yield (Carc\%), fillet yield (Fil\%) and headless gutted carcass yield (HGCarc\%) already reported by Haffray et al. (2012b) are presented Table 1. HGCarc\% was preferred to Fil\% to express fillet yield as it exhibited very high genetic correlation with 
fillet yield $(0.97 \pm 0.01)$ and a higher heritability $(0.54 \pm 0.04$ vs $0.35 \pm 0.04)$ making indirect selection on this trait more efficient than direct selection on fillet yield (see Haffray et al., 2012b).

\subsection{External and internal body shape traits recording}

Each individual was photographed using a digital camera (Canon PowerShot S50 $2592 \times 1944$ pixels) at processing in Nantes. Coordinates of nineteen morphometric points (landmarks) were digitized using Visilog 6.7 software (Figure 1a). Theses coordinates allowed calculating twenty three variables (Figure $1 \mathrm{~b}$ to $1 \mathrm{~d}$ ): seven heights ( $\mathrm{Ht}$ and $\mathrm{H} 1$ to $\mathrm{H} 6$ ), the head perimeter $(P)$, seven lengths (L1 to $L 7)$, six surfaces ( $A 1$ to $A 6)$ and two angles ( $\beta 1$ and $\beta 2)$. The distances between two landmarks $A\left(x_{A}, y_{A}\right)$ and $B\left(x_{B}, y_{B}\right)$ were calculated by $d=\sqrt{\left(x_{B}-x_{A}\right)^{2}+\left(y_{B}-y_{A}\right)^{2}}$. Surfaces were calculated based on angle estimation with AlKashi theorem in a triangle between three landmarks $A, B$ and $C$ for which $A B^{2}=A C^{2}+B C^{2}-$ 2.AC.BC. $\cos (A \hat{C} B)$. Each of the six surfaces was first divided in two triangles for which heights were also estimated using Al-Kashi theorem. Then surface of each triangle was calculated, as Surface $=$ (base ${ }^{*}$ height) $/ 2$, and added to get the total surface of each two triangles.

Four thicknesses and one internal depth measured with ultrasound imagery (Hospimedi, LC100, 7.5 MHz) were represented in Figure 2: Echo4, Echo5, Echo8, Echo11 and Echo 23.

Eighty four new variables were calculated by the division of the previously measured twenty eight variables by BW, BL and LogBW. Twenty one additional variables which we considered as having some biological meaning were also calculated as differences between sums, subtraction and ratios of the calculated variables. The total number of variables was one hundred twenty eight. Only variables with significant effect in the model to predict processing yields were kept for the publication.

\subsection{Selection of variables and definition of models to predict yields}

Among the high number of variables used to describe the fish morphology, a challenging task was to find the best ones to predict yields. Geometric morphometric is a powerful statistic method used to describe and analysis shape changes (Rohlf and Markus, 1993). In order to identify the morphology associated to high or low processing yields, two groups of 100 fish each with the $10 \%$ highest or the $10 \%$ lowest yields were randomly chosen with no significant difference in mean BW.

Shape variations of these 200 fishes were analysed using APS software (Penin, 2007). Briefly the method consists to a Procrustes superimposition of landmarks. The principal components of shape (PCs) are computed from a principal component analysis of the Procrustes residuals. Finally the low and high yields groups were defined in the overall population and a discriminant analysis was performed to obtain the shape vector which discriminates the two groups. The statistical tests were calculated with a reduced number of PCs corresponding to the high value of $\mathrm{R}^{2}$ and F-test. APS allows a graphical visualization of the discriminant function, the shape differences obtain along the discriminant vector contribute and thus contributing to identify pertinent variables to introduce in a prediction model.

Finally these variables were used in a multiple linear regression (Reg.best, $R$ software package) to find the best prediction model with highest $\mathrm{R}^{2}$ and $\mathrm{F}$ value to predict Carc\% (ModCarc\%), Head\% (ModHead\%) and HGCarc\% (ModHGCarc\%). 


\subsection{Validation of the models}

Models were validated by the cross validation technique, for assessing how the results will generalize to an independent data. First the dataset is divided into $K$ subsets, and then the analysis is performed on the data of the $K-1$ subsets called "training sets" and validated on the data of the left apart set, called the "validation set". Multiple rounds are performed and each set is used for training and for validation.

The validation result is The Root Mean Square Error of Prediction (RMSEP). It is calculated at each round and final result is RMSEP averaged over the rounds.

Cross validation was realised with $\mathrm{cv} . \mathrm{glm}$ function of the Boot packages in $\mathrm{R}$ software with $K=20$.

\subsection{Statistical analysis and estimation of genetic parameters}

Mean and standard deviation for each trait and fixed effects were estimated using the SAS software package. Maternal common environment effect, with dams considered as a random effect $(n=60)$, and fixed effects (day of slaughtering, day of measurement) were not significant for the traits considered (Haffray et al., 2012b).

Heritability was estimated using VCE 6 software (Groeneveld et al., 2008) with a univariate animal model for each trait:

$$
Y=\mu+Z a+\varepsilon
$$

where $Y$ is the vector of observations, $\mu$ the mean of the performance, $Z$ the incidence matrice, $a$ the vector of random additive genetic effects and $\varepsilon$ the vector of random residual effects. Genetic correlations between traits were estimated with bivariate animal models.

The expected genetic gains of full-sib selections (FS) or indirect individual selection (IS) on the models were compared to direct individual mass selection (MS), which is a theoretical point since this is not possible to carry out a mass selection on lethal traits, using Falconer and McKay (1996) formulas:

- Expected genetic gain $(\Delta G)$ in the case of direct individual mass selection (MS) was calculated as following: $\Delta G=i h^{2} \sigma_{x}$ where $h^{2}{ }_{x}$ is the heritability of the trait $X, i$ is the selection intensity on $X$ and $\sigma_{x}$ is the phenotypic standard deviation of the trait $X$ selected.

- Expected correlated response (CR) to indirect individual selection (IS) on the models (criteria) was calculated as following: $C R=i_{x} \mathrm{~h}_{x} \sigma_{y} h_{y} r_{g}$ where $i$ is the selection intensity, $h_{x}$ and $h_{y}$ are the square roots of heritability of the trait $X$ selected and $Y$ the trait targeted for improvement, $\sigma_{y}$ is the phenotypic standard deviation of the correlated trait to improve and $r_{g}$ is the genetic correlation between the traits $X$ and $Y$.

- Expected genetic gain in the case of full-sib family selection (FS) was calculated as $R_{s}=\frac{i \sigma_{p} h^{2} n r}{\sqrt{n(1+(n-1) t)}}$, where $i$ is the selection intensity, $\sigma_{\mathrm{p}}$ is the phenotypic standard deviation of the trait selected, $h^{2}$ is its heritability, $n$ is the number of sibs measured per family, $r$ is the correlation between additive genetic values of sibs (here $r=0.5$ within full-sib families) and $t$ is the phenotypic intra class correlation( with $t=r h^{2}$, assuming common environmental effects $\left(\mathrm{c}^{2}\right)$ to be zero as all fish are reared in common garden conditions). Selection methods (MS, FS, IS) were computed with 10 $\%$ selection pressure $(i=1.755)$ on the selected trait or indirect criteria for the three yields to improve. As family selection efficiency depends on the number of sibs measured per family and on between family selection pressure, two scenarii of full-sib 
selection (FS) were calculated with $10 \%$ selection pressure and 100 or 10 sibs per family (FS(10 \%, 100); FS(10\%, 10)). However, as selection efficiency depends also on economic constraints, a third scenario of sib-selection more in agreement with technical and economical practices (see Gjerde et al., 2012; $i=0.47$ to 0.98 ) was also considered with $40 \%$ selection pressure $(i=0.966)$ and 10 sibs per family (FS(40\%, 10)).

\section{Results}

\subsection{Morphology of low and high processing yields rainbow trout}

The graphical visualization of shape differences obtained with the discriminant function for low and high yield fish for Carc\%, Head\% and HGCarc\% were represented on Figure 3.

The discriminant functions were highly significant with three $\mathrm{PCs}$ for $\operatorname{Carc} \%\left(\mathrm{R}^{2}=0.37\right.$ and $\mathrm{F}$ $\left.=38.5, P<10^{-6}\right)$, Head\% $\left(\mathrm{R}^{2}=0.5, \mathrm{~F}=197, P<10^{-6}\right)$ and HGCarc\% $\left(\mathrm{R}^{2}=0.19, \mathrm{~F}=\right.$ 23.5, $\left.P<10^{-6}\right)$.

The graphical visualization of shape with the highest Carc\% was more oblong (or salmon like). The visualization with highest Carc\% exhibited nearly equal dorsal and ventral heights above and under the lateral line. Fish with the lowest Carc\% exhibited higher belly heights than dorsal heights. The anal fin of the fish with the highest Carc\% was advanced (due to smaller belly cavity) and their tail began after the tail of the fish with the lowest Carc\%. The highest Carc\% also corresponded to a smaller head angle than the lower ones.

The two groups differed mainly by their relative head development, the group with the highest $\mathrm{Head \%}$ having a higher head surface, a smaller trunk and a higher head height and length.

The conformation corresponding to the highest HGCarc\% was the same as the conformation for the fish with the highest Carc\% but with a smaller head development.

The variables selected in the models for the three prediction equations are presented in Table 2.

Carc\% could be predicted with three variables (X1, X2 and $\mathrm{X} 3$ ) that associated the homogeneity of body shape from the middle part of the fish to its tail (X1) and the two ratios of abdominal fillet thickness to the development of the belly depth (X2) and the visceral surface reported to the complementary surface of the whole body (X3).

Head \% could be predicted with four variables (X4, X5, X6 and X7) that associated the head surface (A1) reported to the fish thickness (E5) at constant BW, the head height reported to the total body length measures as the total lateral line length (L5 + L6), the condition coefficient $\mathrm{K}$ and the head surface reported to BW.

HGCarc\% could be predicted with the five variables that explained the best Carc\% (X1, X2 and X3) and inversely Head\% (X4; X5 and X7).

The mean predicted values were $88.39 \% \pm 0.99$ for ModCarc\%, $14.17 \% \pm 0.90$ for ModHead\% and $72.38 \% \pm 1.14$ for ModHGCarc\%. 
The phenotypic correlations between yields predicted by the models and real yields to predict calculated from the whole data set ranged from 0.56 to 0.68 (Table 4).

RMSEPs of the models were respectively 1.07 for Carc\%, 0.64 for Head\%, 1.18 for HGCarc\%. These values are systematically inferiors to the standard errors of the values measured for these parameters $(\operatorname{sd}(\operatorname{Carc} \%)=1.4, \operatorname{sd}($ Head\% $)=0.9, \operatorname{sd}(H G C a r c \%)=1.5)$.

\subsection{Heritabilities and genetic correlations}

Heritabilities of the morphological traits measured (lengths, heights, ultrasound measures, surfaces and angles) are represented in Figure 4. Heritabilities were intermediate and ranged from $0.26 \pm 0.05$ (L6) to $0.46 \pm 0.06(\mathrm{~L} 6)$, excepted L3 $(0.05 \pm 0.02)$ and the angle $\beta 2(0.12 \pm$ $0.03)$

Heritabilities of the composite variables $X 1$ to $X 7$ and their genetic and phenotypic correlations with the yields to predict are given in Table 2. The heritabilities were lower (0.230.33) than those of the traits to predict (Table 3).

Heritabilities of the predicted yields (ModCarc\%, ModHead\% and ModHGCarc\%) ranged from 0.25 to 0.28 (Table 3).

Several variables of the models exhibited high genetic correlation with yields to predict or between them: X2 and X3 with Carc\% (0.85 and -0.75), X4 and X7 with Head\% (0.80 and 0.85), X2 with HGCarc\% (0.72), X4 with X7 (0.96 \pm 0.01$)$.

The prediction models tended to present higher genetic correlations with growth and conformation (BW, BL, K) than the real yields to predict (e.g. $-0.68 \pm 0.07$ between Mod Head\% and BW vs $-0.51 \pm 0.10$ between Head\% and BW).

Each predicted yield was highly genetically correlated with real yield : $0.88 \pm 0.04$ for ModCarc\%, $0.90 \pm 0.03$ for ModHead\% and $0.87 \pm 0.05$ for ModHGCarc\%.

\subsection{Comparative expected genetic gains}

Sib-selection with 100 or 10 sibs per full-sibs family [FS(10\%, 100); FS(10\%, 10)] created the same range of genetic progress for a $10 \%$ selection pressure (Figure 5 ). The genetic gains of FS $(10 \%, 10)$ were slightly lower when compared to MS selection: $88.3 \%, 89.6 \%$ and $84.8 \%$ for $\mathrm{Carc} \%$, Head\% and HGCarc\%. FS $(40 \%, 10)$ selection with $40 \%$ selection pressure decreased genetic gain to $50.1 \%, 50.8 \%$ and $48.1 \%$ of theoretical MS selection efficiency.

IS selection progress for Car\%, Head\% and HGCarc\% was $66.5 \%, 66.9 \%$ and $58.7 \%$ of the theoretical progress that could be achieved by MS selection but IS selection was $32.7 \%$, $31.7 \%$ and $21.9 \%$ more efficient than $\mathrm{FS}(40 \%, 10)$ for same targeted processing yields.

\section{Discussion}

The study investigated a new procedure to identify external and/or internal morphological variables phenotypically correlated with different processing yields in large rainbow trout. The application of geometric morphometrics tools provided a visual support to better understand phenotypic differences between high and low yields and to define pertinent combinations between variables to objective differences observed intuitively by human eyes. These 
variables were combined in a linear regression model. Heritabilities of indirect criteria were estimated and the relative efficiency of indirect selection (IS) on these indirect criteria was compared to theoretical mass selection (MS) or sib-selection (FS) with different family sizes (10 or 100$)$ and two different selection pressures (10\% or $40 \%)$.

\subsection{Phenotypic linear models}

Phenotypic correlations between predicted yields and real yields were intermediate $(0.56$ 0.68). A limited number of studies had reported the application of linear combinations of morphological variables to predict phenotypic processing yields in fish (Bosworth et al., 2001; Rutten et al., 2004; Van Sang et al., 2009). The present study estimated 0.56 as correlation coefficient between HGCarc\% (highly correlated with Fil\%), estimation in the range of prediction of fillet yield by previous authors: 0.16 to 0.50 in females and males catfish (Bosworth et al., 2001), 0.38 in Nile tilapia (Rutten et al., 2004) and 0.77 in river catfish (Van Sang et al., 2008). None of them investigated Carc\% or Head\% and results on these last two traits cannot be compared with previous work. The values estimated for RMSEP are quite low and inferior to standard errors, which validate the ability of the models to predict yields with acceptable precision for new data.

Fish with the highest Carc\% had a homogeneous antero-posterior body shape, a smaller visceral depth combined to a high abdominal muscle thickness and a smaller relative visceral surface. Fish with the lowest Head\% were those with a smaller head surface and height and the highest body thickness. Those with the highest HGCarc\%, and therefore fillet yield, were fish that combined traits defined in the previous two models. These results confirmed that differences in body shape can be phenotypically link to muscle and adipose tissue development and associated with processing yields in fish (Bosworth et al., 2001; Rutten et al., 2004; Van Sang et al., 2008).

\subsection{Genetic parameters of the predicted yields}

Heritabilities of the combined variables that were used for the models were moderate $(0.23-$ $0.33)$, or intermediate for $K(X 6,0.56)$. These moderate values, lower than some of the heritabilities estimated for some traits that composed these variables may be explained by intermediate genetic correlations between these traits and some antagonistic relationship.

The predicted yields exhibited moderate heritabilities $(0.25-0.28)$. The intermediate genetic correlations between the combined traits included in the models could also contribute to these limited heritabilities.

Genetic correlations of predicted yields with real yields were high (0.87-0.90). Some of the variables that composed the models exhibited very high genetic correlations with the yields to predict (X2 and X3 with Carc\%, X4 and X7 to Head \% and X2 with HGCarc\%) making some of these variables (like $X 2$ ) nearly equally genetically correlated with the trait to predict. However, none of the variables included in the models exhibited as high a genetic correlation as the predicted yields, which were then preferred.

Previous authors that used single morphometric variables (Gjerde and Shaeffer, 1989; Neira et al., 2004; Rutten et al., 2005) or linearly combined variables (Van Sang et al., 2012) did not find added value to these variables when compared to the measurement of the yield to predict on sibs. However, none of them investigated such a large number of variables combining lengths, height and surfaces or body thicknesses or belly depth. 
The variable X2 was highly genetically correlated with Carc\%. X2 is partly composed by Echo8 already reported as moderately genetically correlated (0.46-0.63) with dressing percentage (refered as Carc\% here) by Gjerde and Shaeffer (1989) in rainbow trout. However, the measurement of Echo23, the other component of $\mathrm{X} 2$, was never reported before. X2 is highly genetically correlated $(0.85)$ with Carc\% and is the first variable selected in the Carc\% and HGCarc\% models. The additional external morphometric variable allowed increasing genetic correlations with the model to 0.88 . The gain in genetic correlation is limited when comparing predicted yields to X2. The predicted yield added information on different relative body compartments and integrated other sources of morphological variations than $\mathrm{X} 2$.

Curiously, fillet thickness at different locations (Echo4 and Echo5) were not selected in ModHGCarc\% that is highly genetically correlated with $\mathrm{HGCarc \%}$ and highly genetically correlated to Fil\% (0.87). As also proposed by Kause et al. (2004), the visceral fat deposit variation may mostly determine $H G C a r c \%$ variation, and higher here than fillet thickness.

The present study confirmed the potential of ultrasound tomography as initially investigated phenotypically in the American catfish (Bosworth et al., 2001). This technology is routinely used in livestock breeding programs to predict backfat thickness and increase percentage of lean cuts in pig (Lo et al., 1992), backfat thickness and longissimus muscle area or lean meat yield in beef cattle (Crew and Kemp, 2001; Nalaila et al., 2012) or breast meat yield in broiler and turkey (Gaya et al., 2006; Case et al., 2011). The genetic correlations estimated with the yield to predict $(0.87-0.90)$ in this study are in the same range as the trait measured to predict longissimus muscle area (0.87) in pig (Lo et al., 1992) and much higher than the trait measured to predict breast meat yield (0.64-0.69) in broiler (Gaya et al., 2006) or (0.47-0.75) in turkey (Case et al., 2012). When compared to Gjerde and Shaeffer (1989), Rye and Refstie (1995) and Neira et al. (2004) that measured morphological traits with calipers on slaughtered fish, ultrasounds constitutes a major advancement in fish breeding as the trait can be measured on live candidates.

\subsection{Relative genetic gains}

Whatever the numbers of sibs measured per family, FS selection create same range of genetic progress at the same selection pressure. Advantage of FS selection is its simplicity as no other measures than the processing yields have to be collected.

IS selection created less genetic progress than FS selection at the same selection pressure even if models are highly correlated with the yields to predict. This was associated with the two times lower heritability of the models. This is also associated with the better efficiency of FS selection when the family size is higher than 10.

However, when selection pressure applied to FS selection was decreased to more realistic and economic selection pressure [FS $(40 \%, 10)]$ as reported by Gjerde et al. (2012), the relative efficiency of FS selection drop under the efficiency of IS selection kept at $10 \%$ selection pressure (IS, 10\%). These highest selection responses benefits from the very high genetic correlation between the models and the yields to predict, these high genetic correlations having a more important impact in the estimation of the response than the nearly $50 \%$ lowest heritability of the models than the yields to predict.

However, very low correlated responses may also be expected with IS selection when traits heritability is very limited or close to zero as for fillet yield in some experiments (Rutten et al., 2005; Powel et al., 2009; Nguyen et al., 2010; Gjerde et al., 2012; Van Sang et al., 2012). 
The cost to measure traits in FS selection schemes (rearing costs of additional full-sibs, labour and financial loss of the carcasses) has to be balanced with the cost to measure the traits to be combined in the models for IS selection. Whatever the selection pressure applied by breeders, a technical limitation of IS selection on models is the time to collect the morphometric coordinates (here 1.5 month for nearly 2000 fish, without data treatment). $A$ posteriori data collection from digitalized pictures, as done in this study, is applicable by breeding companies. The increase of selection pressure on models will imply to increase the number of fish measured. This should require the development of adapted 2D or 3D imaging software before field applications. Marty-Mahé et al. (2004) and Blonk et al. (2010) provided first basis of automation of picture and body size traits captures by digital image analyse in fish.

\subsection{Uncertainty of selection on ratios}

The genetic parameters estimated need to be taken with caution as they are composed of ratios or combinations of ratios. Theoretical bases of genetic parameters and selection on ratios is an unresolved subject (Sutherland, 1965) even if selection on condition coefficient K (that is a ratio) was efficient in fish (Ankorion et al., 1992). Pure mathematical combinations between the relative variations of the ratio compounds and body weight, their relative heritability and their high genetic correlations with body weight make results of such selection difficult to achieve. Several authors (Rutten et al., 2005; Nguyen et al., 2010; Gjerde et al., 2012) suggested preferring selection on body weight to increase the flesh quantity. But selection on body weight failed to improved fillet yield (Bonnet et al., 2002; Vandeputte et al., 2009; Nguyen et al., 2010). Combined selection on body weight and fillet yield (3 generations) also failed to increase fillet yield in tilapia (Gjerde et al., 2012), which is probably linked to the limited heritability of fillet yield $(0.06 \pm 0.04)$ reported in this study. However, the between strain variation of gutted yield in rainbow trout (Morkramer et al., 1985), the positive response to selection on body shape (Ankorion et al., 1992) or processing yields in the channel catfish (Bosworth and Wolters, 2009) provided first insights of potential interest of the selection on body shape, or fillet yield to improve fillet yield as suggested by other authors (Kause et al. 2007; Kocour et al., 2007; Haffray et al., 2007; Saillant et al., 2009; Navarro et al., 2009; Powell et al., 2009; Haffray et al., 2012b). The recent advances in using the residuals of allometric relationship between body compartments and body weight suggested (Haffray et al., 2012) or demonstrated (Egset et al., 2012) that selection on this residual, as used to select of feed efficiency in livestock, can be effective to modify equilibrium between body compartments independently of body weight.

\section{Conclusion and perspective}

This study evaluated a procedure to identify internal and external measures combined in a linear regression model to predict phenotypic processing yields. This study provides a major advancement in fish breeding using real time ultrasound imagery to quickly and noninvasively measure a limited number of body thicknesses highly genetically associated with yields of economic interest.

Estimations of genetic progress to indirect selection on the models provided expectation of improvements due to the very high genetic correlations between prediction from the models developed and their yields to predict but also the higher potential selection pressure on live candidates than with sib family selection. The genetic parameters of these predicted yields are encouraging for application in fish breeding. However, potential mathematical bias 
associated with estimations of heritabilities of ratios and their genetic correlation with other traits make it necessary to estimate realized heritability obtained after a selective breeding on these ratios or on the predicted yields.

\section{References}

Agreste, 2009. La salmoniculture française à l'étiage. Agreste Primeur. 4 p., (In French).

Ankorion, Y., Moav, R., Wohlfarth, G.W., Year? Bidirectionnal mass selection for body shape in common carp. Genet Sel. Evol. 24, 43-52.

Blonk, R.J.W., Komen, J., Tenghe, A., Kamstra, A., van Arendonk, J.A.M., 2010. Heritability of shape in common sole, Solea solea, estimated from image analysis data. Aquaculture 307, 6-11.

Bonnet; S., Haffray; P., Chevassus; B., Aubin; J., Fauconneau; B., 2002. Conformation and carcass quality traits in seawater adult brown trout: correlated responses to selection for freshwater body length growth and triploidy*selection interactions. Aquaculture 204 (3-4); 193. Abstract?

Bosworth, B.G., Holland, M., Brasil, B.L., 2001. Evaluation of ultrasound imagery and body shape to predict carcass and fillet yield in farm-raised catfishes. Journal of Animal Science 79, 1483-1490.

Bosworth, B.G., Wolters, W.R., 2009. Realized heritabilities for meat yield traits in divergently selected lines of channel catfish, Ictalurus punctatus. Aquaculture 247 (1-4), 6. Single page?

Bugeon, J., Lefèvre, F., Cardinal, M., Uyanik, A., Davenel, A., Haffray, P., 2010. Flesh quality in large rainbow trout with high or low fillet yield. J. Muscle Foods 21, 702-721.

Case, L.A., Wood, B.J., Miller, S.P., 2012. The investigation of ultrasound technology to measure breast muscle depth as a correlated trait to breast meat yield in Turkey (Meleagris gallopavo) J. Anim. Sci. 90, 3410-3417.

Crews, D.H.J., Kemp, R.A., 2001. Genetic parameters for ultrasound and carcass measures of yield and quality among replacement and slaughter beef cattle. J. Anim. Sci. 79, 3008-3020.

Dupont-Nivet; M., Vandeputte; M., Haffray; P., Chevassus; B., 2006. Effect of different mating designs on inbreeding, genetic variance and response to selection when applying individual selection in fish breeding programs. Aquaculture 252, 161-170.

Egset, C.K., Hansen, T.F., Le Rouzic, A., Bolstad, G.H., Rosenqvist, G., Pélabon, C., 2012. Artificial selection on allometry: change in elevation but not slope. J. Evol. Biol. 1-11. doi: 10.1111/j.1420-9101.2012.02487.x.

Falconer, D.S., McKay, T.F.C., 1996. Introduction to Quantitative Genetics. Longman, Harlow, UK, 455pp.

Gaya, L.G., Ferraz, J.B., Rezende, F.M., Mourao, G.B., Mattos, E.C., Eler, J.P., Michelan Filho, T., 2006. Heritability and genetic correlation estimates for performance and carcass and body composition traits in a male broiler line. Poult. Sci. 85, 837-843.

Gjerde, B., Shaeffer, L.R., 1989. Body traits in rainbow trout. II. Estimates of heritabilities and phenotypic and genetic correlations. Aquaculture 80, 25-44.

Gjerde, B., Mengistu, S.B., Ødegard, J., Johansen, H., Altamirano, D.S., 2012. Quantitative genetics of body weight, fillet weight and fillet yield in Nile tilapia (Oreochromis niloticus). Aquaculture 342-343, 117-124.

Haffray; P., Pincent; C., Rault, P., Coudurier, B., 2004. Domestication et amélioration génétique des cheptels piscicoles français dans le cadre du SYSAAF. INRA Prod. Anim. 17(3), 243-252 (In French).

Haffray, P., Pincent, C., Dupont-Nivet, M., Vandeputte, M., Merdy, O., Chavanne, H., Chatain, B., 2007. Heritabilities and $G \times E$ interactions for quality traits in the European sea bass (Dicentrarchus labrax L.). Aquaculture 272, S265. 
Haffray, P., Vandeputte, M., Petit, V., Pincent, C., Chatain, B., Chapuis, H., Quillet, Dupont-Nivet, M., 2012a. Minimizing maternal effect in salmonids families mixed since eyed stages for selection and a posteriori DNA-pedigreed. Livest. Sci. 150 (1-3), 170178.

Haffray, P., Pincent, C., Chapuis, H., Mazeiraud, E., Petit, V., Rault, P., Bugeon, J., Chatain, B., Vandeputte, M., Dupont-Nivet, M., 2012b. Negative genetic correlations between production traits and head or bony tissues in large all-female rainbow trout (Oncorhynchus mykiss). Aquaculture 368-369, 145-152.

Havenstein, G.B., Ferket, P.R., Qureshi, M.A., 2003. Carcass composition and yield of 1957 versus 2001 broilers when fed "typical" 1957 and 2001 broiler diets. Poult. Sci. 82, 1509-1518.

Kause, A., Paananen, T., Ritola, O., Koskinen, H., 2007. Direct and indirect selection of visceral lipid weight, fillet weight, and fillet percentage in a rainbow trout breeding program. J. Anim. Sci. 85, 3218-3227.

Knap, P.W., Rauw., W.M., 2009. Selection for high production in pigs. In: Rauw, W.M. (Ed.), Resources Allocation Theory Applied to Farm Animal Production. . Cab International, pp. 210-229.

Kocour, M., Mauger, S., Rodina, M., Gela, D., Linhart, O., Vandeputte, M., 2007. Heritability estimates for processing and quality traits in common carp (Cyprinus carpio L.) using a molecular pedigree. Aquaculture 270, 43-50.

Lo, L.L., Mclaren, D.G., Mckeith, F.K., Fernando, R.L., Novakofski, J., 1992. Genetic analyses of growth, real-time ultrasound, carcass, and pork quality traits in Duroc and landrace pigs: II. Heritabilities and correlations. J. Anim. Sci. 70, 2387-2396.

Marty-Mahe P., Loisel P., Fauconneau B., Haffray P., Brossard D. and Davenel A., 2004. Quality traits of brown trout Salmo trutta cutlets described by automated color image analysis. Aquaculture, 232, 225-240.

Morkramer, S., Hörstgen-Schark, G., Langholz, H.J., 1985. Comparison of different European rainbow trout populations under intensive production conditions. Aquaculture, 44, 303-320.

Nalaila, S.M., Stothard, P., Moore, S.S., Wang, Z., Li, C., 2012. Whole genome fine mapping of quantitative trait loci for ultrasound and carcass merit traits in beef cattle. Can. J. Anim. Sci., 91, 61_73.

Navarro, A., Zamorano, M.J., Hildebrandt, S., Ginés, R., Aguilera, C., Afonso, J.M., 2009. Estimates of heritabilities and genetic correlations for growth and carcass traits in gilthead seabream (Sparus auratus L.), under industrial conditions. Aquaculture 289, 25-230.

Neira, R., Lhorente, J.P., Aranedaı, C., Dıaz, N., Bustos, E., Alert, A., 2004. Studies on carcass quality traits in two populations of Coho salmon (Oncorhynchus kisutch): phenotypic and genetic parameters. Aquaculture 241, 117-131.

Nguyen, H.H., Ponzoni, R.W., Abu-Bakar, K.R., Hamzah, A., Khaw, H.L., Yee, H.Y., 2010. Correlated response in fillet weight and yield to selection for increased harvest weight in genetically improved farmed tilapia (GIFT strain), Oreochromis niloticus. Aquaculture $305,1-5$.

Penin X. 2007. APS. A Procrustes software. http://www.procuste.com/aps.html

Powell, J., White, I., Guy, D., Brotherstone, S., 2009. Genetic parameters of production traits in Atlantic salmon (Salmo salar). Aquaculture 274, 225-231.

Rutten, M. J. M., Bovenhuis, H., Komen, H., 2004. Modelling fillet traits based on body measurements in three Nile tilapia strains (Oreochromis niloticus, L.). Aquaculture, 231, (1-4), 113-122

Rutten J.M., Bovenhuis H., Komen H., 2005, Genetic parameters for fillet traits and body measurements in Nile tilapia (Oreochromis niloticus L.). Aquaculture 246, 125-132.

Sutherland, T.M., 1965. The correlation between feed efficiency and rate of gain, a ratio and its denominator. Biometrics 21, 739-749.

Rohlf, F. J. \& Marcus, L. F. (1993). A revolution in morphometrics. Trends in Ecology and Evolution 8, 129-132. 
Rye, M., Refstie, T., 1995. Phenotypic and genetic parameters of body size traits in Atlantic salmon Salmo salar. Aquaculture Research, 26, 875-885.

Saillant, E.., Dupont-Nivet, M., Sabourault, M., Haffray, P., Laureau S., Vidal, M-O., Chatain, B., 2009. Genetic variation for carcass quality traits in cultured sea bass

Vandeputte, M., Bugeon, J., Dupont-Nivet, M., Chavanne, H., Haffray, P., Lefèvre, F., Labbé, L., Vergnet, A., Chatain, B., 2009. Processing quality of selected fish: quasi-absence of correlated responses to selection on growth in the brown trout Salmop trutta and the sea bass Dicentrarchus labrax. EAS Aquaculture Europe Conference, August 14-17, 2009, 627-628.

Van Sang, N., Thomassen, M., Klemetsdal, G., Gjøen, H. M., 2009. Prediction of fillet weight, fillet yield and fillet fat for live river catfish (Pangasianodon hypophthalmus). Aquaculture, 288, 166-171.

Van Sang, N., Klemetsdal, G., Ødegård, J., Gjøen, H. M., 2012. Genetic parameters of economically important traits recorded at a given age in striped catfish (Pangasianodon hypophthalmus). Aquaculture, 344-349, 82-89

\section{Figures}

Figure 1: Landmarks (1a) (1 to 19) used to calculate: $1 \mathrm{~b}$ ) seven heights ( $\mathrm{Ht}$ and $\mathrm{H} 1$ to $\mathrm{H} 6$ ) and the head perimeter $(P) ; 1 c)$ seven lengths (L1 to L7); $1 d$ ) and 1e) six surfaces (A1 to $A 6)$; $1 \mathrm{e})$ two angles ( $\beta 1$ and $\beta 2)$ on large rainbow trout.

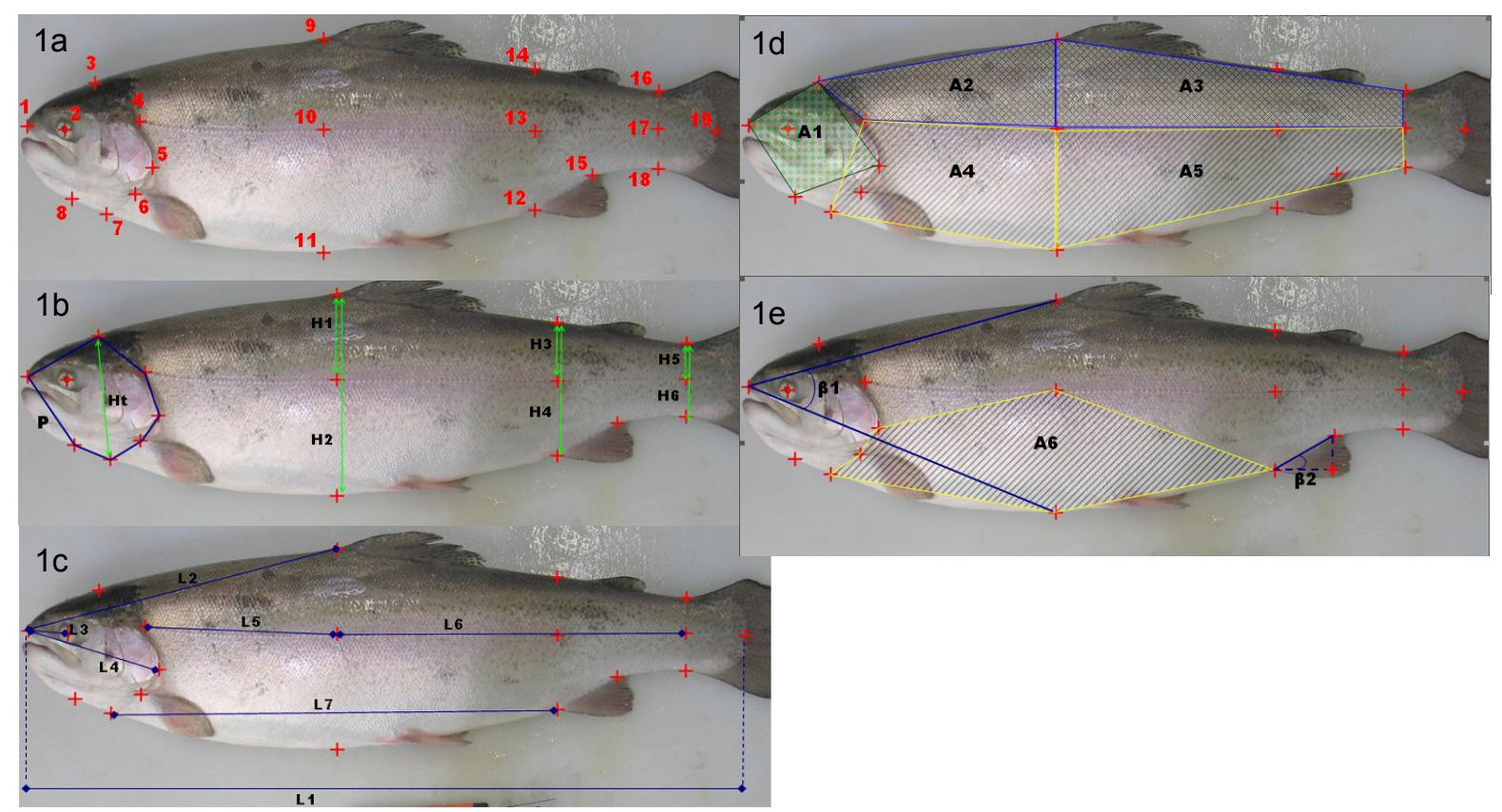


Figure 2: Position of the five Echo4, Echo5, Echo8, Echo11 and Echo23 ultrasound measures $( \pm 0.01 \mathrm{~mm})$ obtained with Hospimedi LC100 ultrasound machine equipped with linear $7.5 \mathrm{MHz}$ probe.

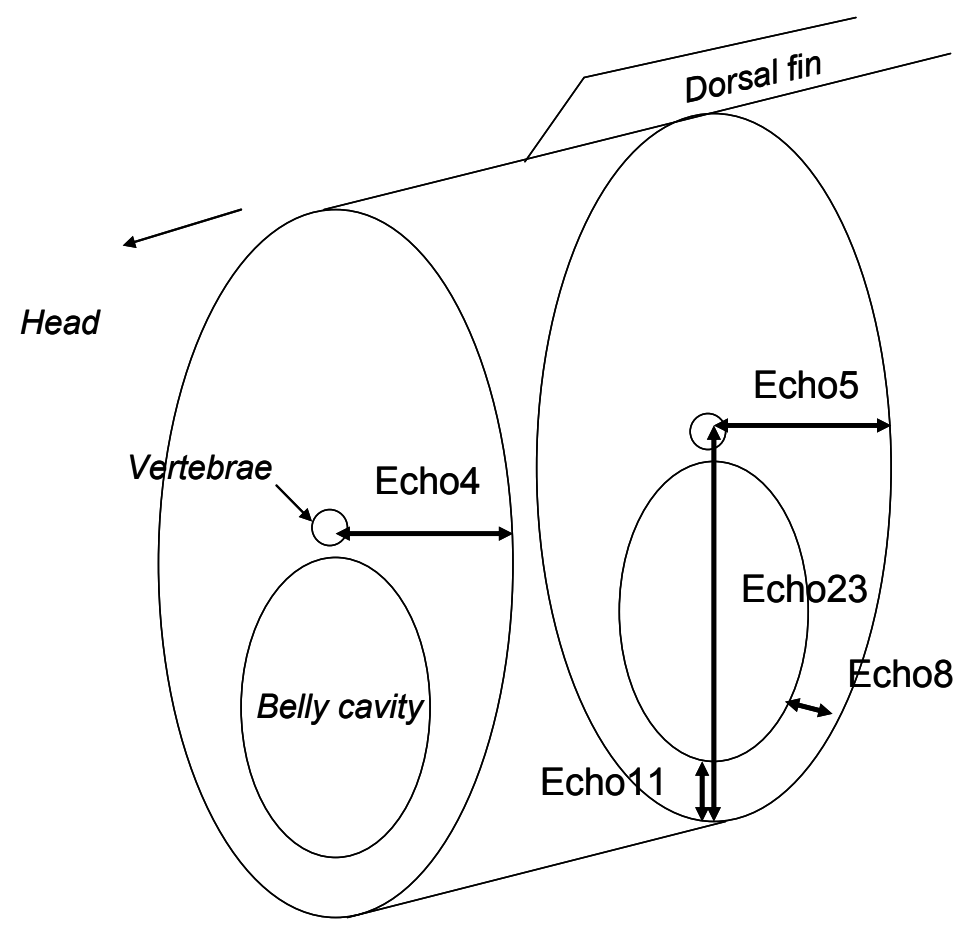


Figure 3: Graphical visualization of shapes differences with the discriminant vectors for Carc\%, Head\% and HGCarc\% using APS software (Penin 2007): 2a) highest Carc\% is represented in red and the lowest in blue; 2b) Highest Head\% is represented in red and the lowest in blue; 2c) Highest HGCarc\% is represented in red and the lowest in blue.
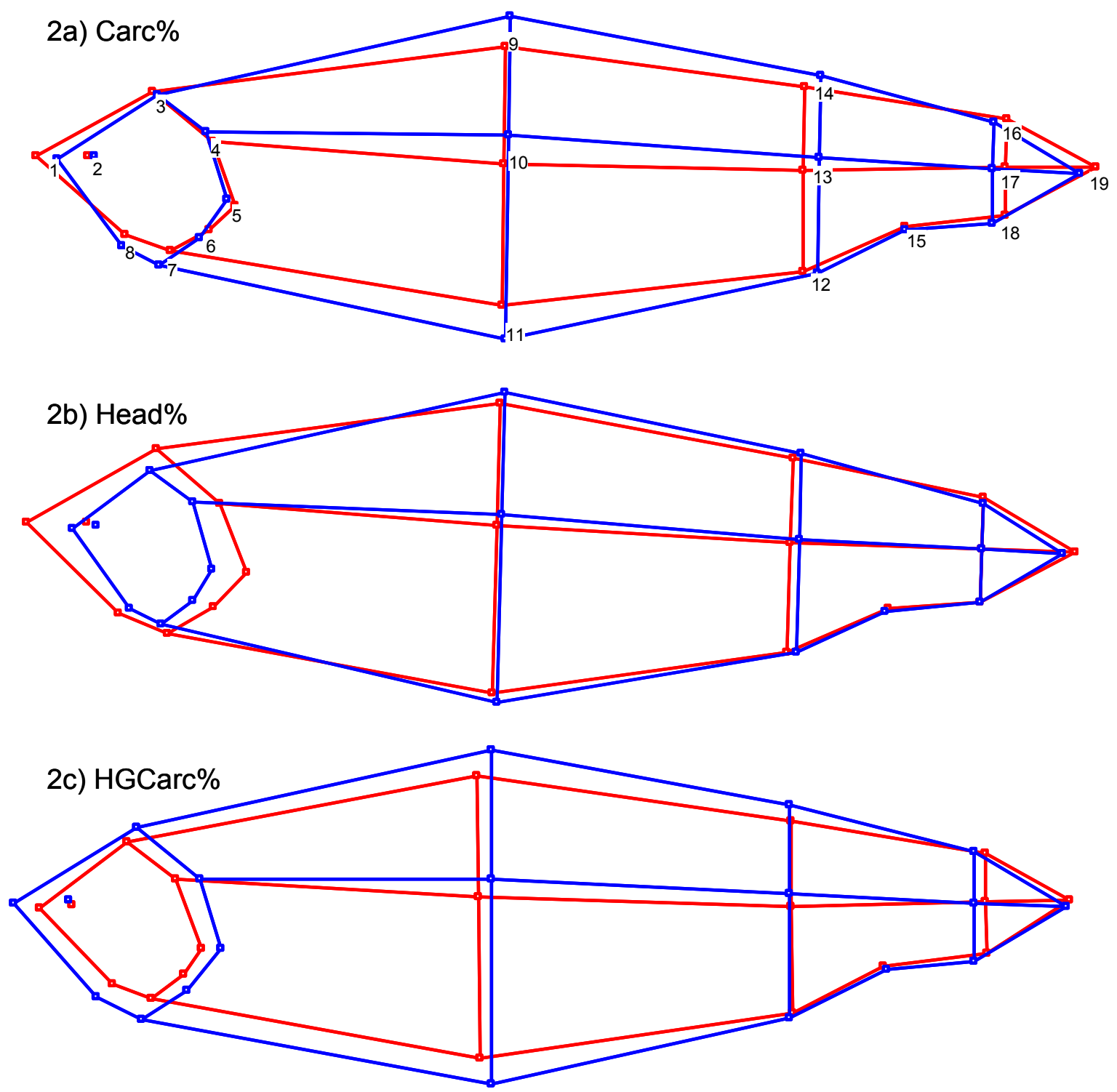
Figure 4a, b, c and d: Heritability of: a) body lengths ( $\mathrm{L} 1$ to $\mathrm{L} 7$; b) body heights ( $\mathrm{Ht}$ and $\mathrm{H} 1$ to $\mathrm{H6}$ ); c) ultrasound measures (Echo4 to Echo23) and d) surface (A1 to A6) and angles ( $\beta 1$ and $\beta 2$ ). Error bars: standard errors.
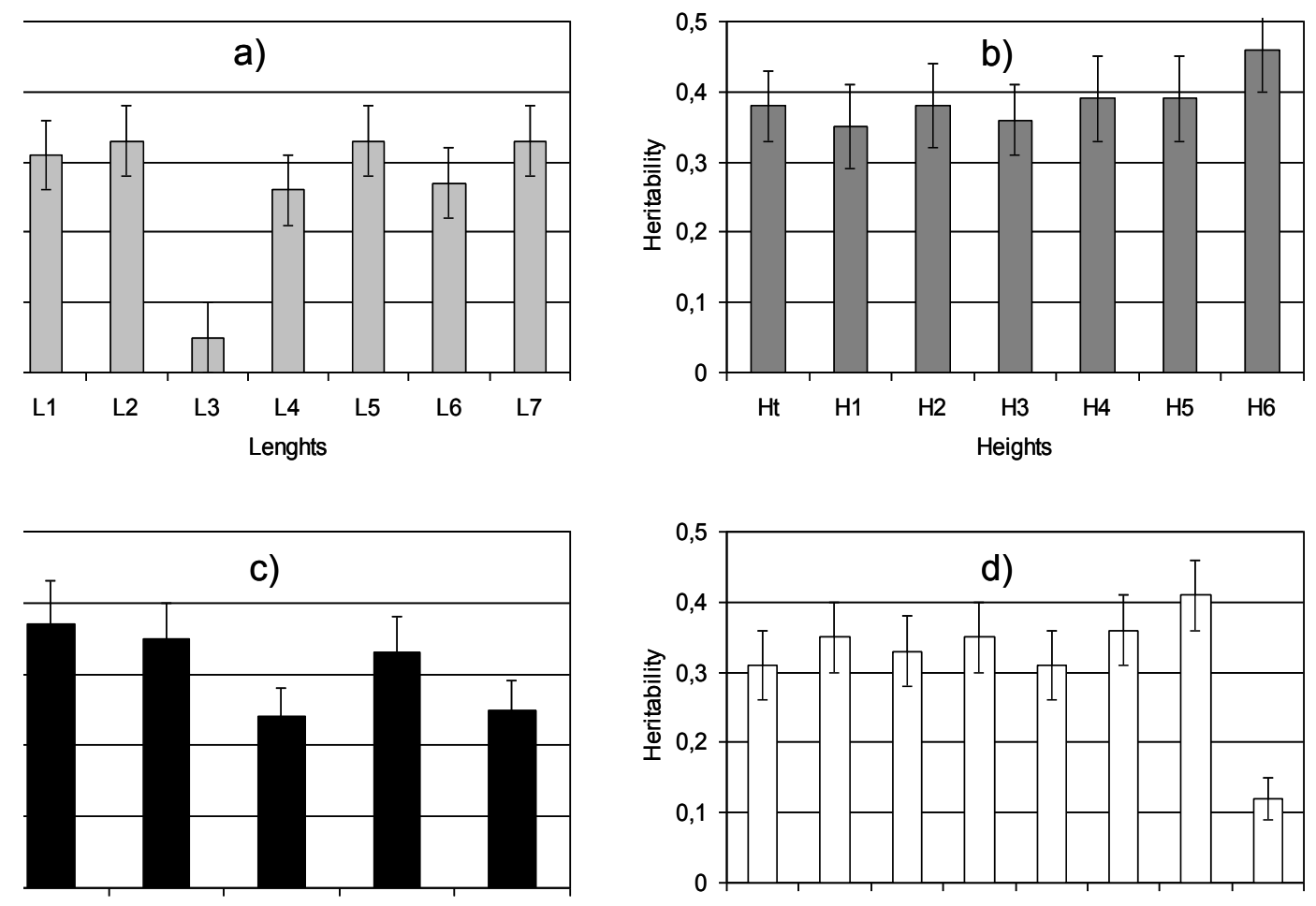
Figure 5: Theoretical genetic gain (\%) per generation on carcass yield (Carc\%), head yield (Head\%) and headed and gutted carcass yield (HGCarc\%) according to five strategies of selection: $10 \%$ selection pressure when selection is done on the trait by theoretical mass selection (MS(10\%)), when the trait is family selected with 100 or 10 full sibs measured per family (FS $(10 \%, 100)$ or $\mathrm{FS}(10 \%, 10))$, when indirect selection on morphological models is applied on live candidates (IS, 10\%)), or with $40 \%$ selection pressure with 10 full sibs per family (FS $(40 \%, 10))$.

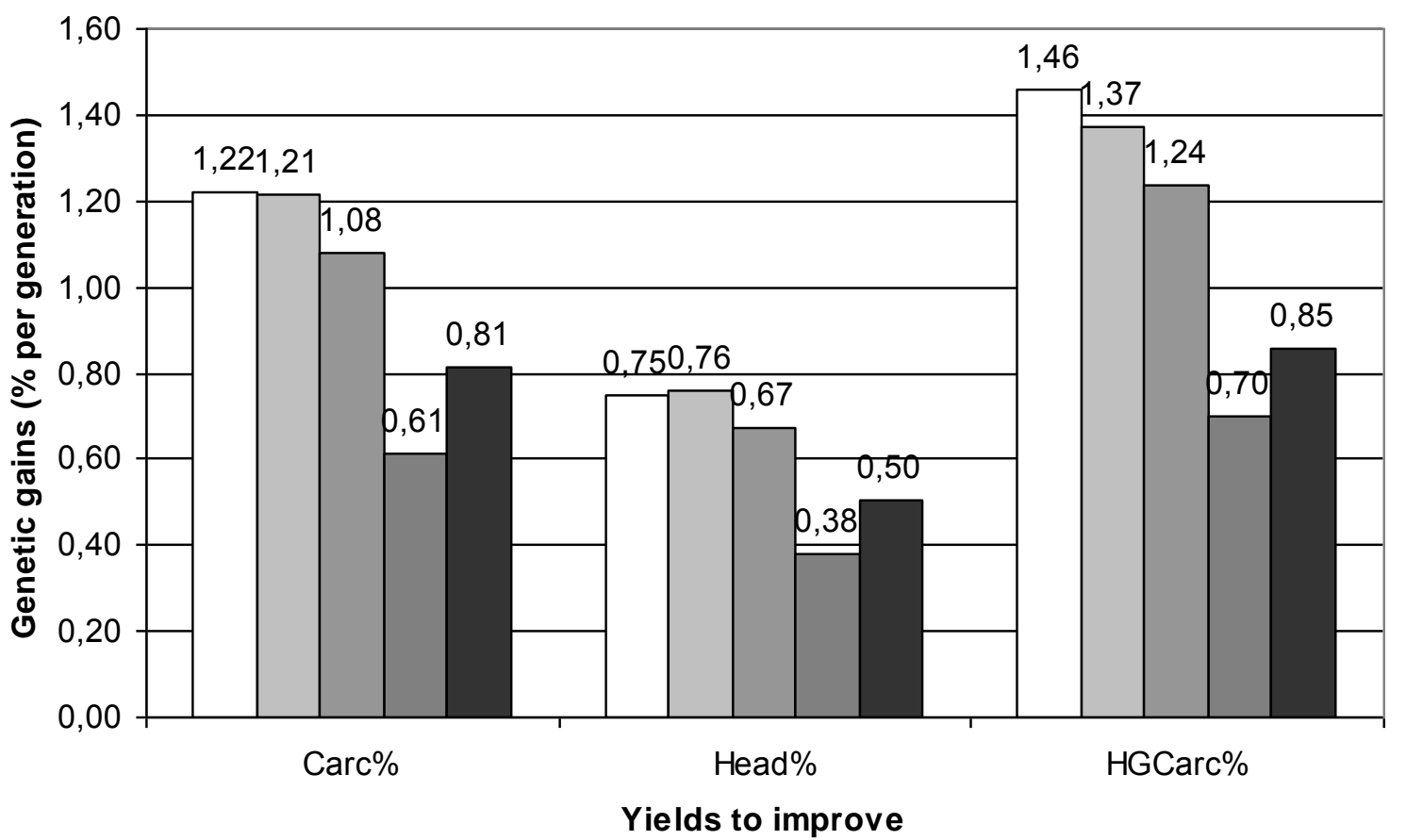

$\square \mathrm{MS} 10 \% \square \mathrm{FS}(10 \% ; 100) \square \mathrm{FS}(10 \% ; 10) \square \mathrm{FS}(40 \% ; 10) \square \mathrm{IS}(10 \%)$

\section{Tables}

Table 1: Mean \pm standard deviation, maximum, minimum and number of rainbow trout Oncorhynchus mykiss measured (N) in for body weight (BW), body length (BL), condition coefficient $(\mathrm{K})$, carcass yield (Carc\%), head yield (Head\%), headless gutted carcass yield (HGCarac\%).

\begin{tabular}{|l|l|l|l|l|}
\hline Trait & Mean \pm SD & Minimum & Maximum & $\mathrm{N}$ \\
\hline BW $(\mathrm{g})$ & $1639.2 \pm 350.9$ & 424.5 & 2801.5 & 1962 \\
\hline $\mathrm{BL}(\mathrm{cm})$ & $48.1 \pm 3.0$ & 36.2 & 55.2 & 1958 \\
\hline $\mathrm{K}\left(10{ }^{*} \mathrm{~g} / \mathrm{cm}^{3}\right)$ & $1.46 \pm 0.11$ & 1.08 & 1.93 & 1935 \\
\hline Carc\% & $87.7 \pm 1.4$ & 82.3 & 93.4 & 1948 \\
\hline Head\% & $11.1 \pm 0.9$ & 8.3 & 14.9 & 1925 \\
\hline HGCarc\% & $76.6 \pm 1.5$ & 71.0 & 81.4 & 1913 \\
\hline
\end{tabular}


Table 2: Multiple linear regression models to predicted carcass yield (ModCarc\%), head yield (ModHead\%) and headed and gutted carcass yield (ModHGCarc\%) in rainbow trout with $\mathrm{R}^{2}$,F Fisher test value, RMSEP and prediction equation.

Carc\%

\begin{tabular}{|l|c|l|}
\hline $\mathrm{X} 1$ & $(\mathrm{H} 4-\mathrm{H} 3) /(\mathrm{H} 2-\mathrm{H} 1)$ & Ratio between ventral heights \\
$\mathrm{X} 2$ & Echo8/Echo23 & Ratio between belly ultrasound measures \\
$\mathrm{X} 3$ & $\mathrm{~A} 6 /(\mathrm{A} 1+\mathrm{A} 2+\mathrm{A} 3+\mathrm{A} 4+\mathrm{A} 5)$ & Ratio between belly surface and the total body surface \\
\hline \multicolumn{2}{|c|}{ Regression characteristics: $\mathrm{R}^{2}: 0,40 \quad \mathrm{~F}: 400,7 \quad$ RMSEP $=1.07$} \\
\hline \multicolumn{2}{|c|}{ ModCarc\% $=88,6607+7,5733 . \mathrm{X} 1+32,8458 . \mathrm{X} 2-26.1840 . \mathrm{X} 3$}
\end{tabular}

Head \%

\begin{tabular}{|ccl|}
\hline & & Ratio between head surface and body thickness measured by \\
X4 & $(\mathrm{A} 1 / \mathrm{E} 5) / \mathrm{BW}$ & ultrasound reported to the total body weight \\
$\mathrm{X} 5$ & $\mathrm{Ht} /(\mathrm{L} 5+\mathrm{L} 6)$ & Ratio between head height and lateral line length \\
$\mathrm{X} 6$ & $\mathrm{~K}$ & Fulton coefficient \\
$\mathrm{X} 7$ & $\mathrm{~A} 1 / \mathrm{BW}$ & Ration between head surface and body weight \\
\hline \multicolumn{4}{|c|}{ Regression characteristics : $\mathrm{R}^{2}: 0,50 \quad \mathrm{~F}: 440,6 \quad \mathrm{RMSEP}=0.64$} \\
\hline
\end{tabular}
ModHead\% = 9,21682 - 0,30296.X4 + 19,90169.X5 - 2,17887.X6 + 1,97149.X7

HGCarc\%

\begin{tabular}{|ccl|}
\hline X2 & Echo8/Echo23 & Ratio between belly ultrasound measures \\
X1 & $(\mathrm{H} 4-\mathrm{H} 3) /(\mathrm{H} 2-\mathrm{H} 1)$ & Ratio between ventral heights \\
$\mathrm{X} 3$ & $\mathrm{~A} 6 /(\mathrm{A} 1+\mathrm{A} 2+\mathrm{A} 3+\mathrm{A} 4+\mathrm{A} 5)$ & Ratio between belly surface and the total body surface \\
& & Ratio between head surface and body thickness measured by \\
$\mathrm{X} 4$ & $(\mathrm{~A} 1 / \mathrm{E} 5) / \mathrm{BW}$ & ultrasound reported to the total body weight \\
$\mathrm{X} 5$ & $\mathrm{Ht} /(\mathrm{L} 5+\mathrm{L} 6)$ & Ratio between head height and lateral line length \\
$\mathrm{X} 7$ & $\mathrm{~A} 1 / \mathrm{BW}$ & Ration between head surface and body weight \\
\hline & Regression characteristics: $\mathrm{R}^{2}: 0,38 \quad \mathrm{~F}: 179 \quad$ RMSEP=1.18 \\
\hline
\end{tabular}

ModHGCarc\% $=84,47835+30,57464 . X 2+4,38170 . X 1-26,00902 . X 3+0,47883 . X 4$ $17,75211 . X 5-3,01471 . X 7$ 
Table 3: Heritability of selected traits included in the three different models to predict carcass yield (Carc\%), head yield (Head\%) and headed and gutted carcass yield (HGCarac\%) and their genetic correlations with body weight (BW), body length (BL) and the yields to predict: carcass yield (Carc\%), head yield (Head\%) and headed and gutted carcass yield (HGCarc\%)

\begin{tabular}{|c|c|c|c|c|c|c|c|}
\hline & $\mathrm{X} 1$ & $\mathrm{X} 2$ & $\mathrm{X} 3$ & $\mathrm{X} 4$ & $\mathrm{X} 5$ & X6 & $\mathrm{X7}$ \\
\hline Trait & $\begin{array}{l}(\mathrm{H} 4-\mathrm{H} 3) / \\
(\mathrm{H} 2-\mathrm{H} 1)\end{array}$ & Echo8/Echo23 & $\begin{array}{c}\mathrm{A} 6 / \\
(\mathrm{A} 1+\mathrm{A} 2+\mathrm{A} 3+\mathrm{A} 4+\mathrm{A} 5)\end{array}$ & $(\mathrm{A} 1 / \mathrm{E} 5) / \mathrm{BW}$ & $\begin{array}{c}\mathrm{Ht} / \\
(\mathrm{L} 5+\mathrm{L} 6)\end{array}$ & $\mathrm{K}$ & $\mathrm{A} 1 / \mathrm{BW}$ \\
\hline Heritability & $0.23 \pm 0.04$ & $0.24 \pm 0.04$ & $0.25 \pm 0.04$ & $0.31 \pm 0.05$ & $0.33 \pm 0.05$ & $0.54 \pm 0.06$ & $0.26 \pm 0.05$ \\
\hline rg BW & $-0.26 \pm 0.11$ & $-0.14 \pm 0.13$ & $0.29 \pm 0.12$ & $-0.86 \pm 0.03$ & $0.21 \pm 0.12$ & $0.71 \pm 0.06$ & $-0.70 \pm 0.07$ \\
\hline rg BL & $-0.13 \pm 0.13$ & $-0.13 \pm 0.13$ & $0.11 \pm 0.13$ & $-0.72 \pm 0.06$ & $0.02 \pm 0.12$ & $0.34 \pm 0.10$ & $-0.55 \pm 0.09$ \\
\hline rg $\mathrm{Carc} \%$ & $0.63 \pm 0.08$ & $0.85 \pm 0.05$ & $-0.75 \pm 0.06$ & $0.22 \pm 0.11$ & $0.01 \pm 0.11$ & $-0.13 \pm 0.09$ & $0.30 \pm 0.11$ \\
\hline rg Head\% & $0.37 \pm 0.11$ & $0.12 \pm 0.13$ & $-0.18 \pm 0.12$ & $0.80 \pm 0.05$ & $0.48 \pm 0.09$ & $-0.47 \pm 0.08$ & $0.85 \pm 0.04$ \\
\hline $\operatorname{rg~HGCarc\% }$ & $0.40 \pm 0.11$ & $0.72 \pm 0.07$ & $-0.59 \pm 0.09$ & $-0.23 \pm 0.11$ & $-0.27 \pm 0.11$ & $0.13 \pm 0.10$ & $-0.21 \pm 0.12$ \\
\hline
\end{tabular}


Table 4: Heritability ( \pm standard error) estimates (diagonal), phenotypic correlations (below the diagonal) and genetic correlations \pm standard error (above the diagonal) in rainbow trout Oncorhynchus mykiss for body weight (BW), body length (BL), condition coefficient (K), carcass yield (Carc\%), head yield (Head\%), headless gutted carcass yield (HGCarc\%) already published (Haffray et al., 2012b) and models of prediction of carcass yield (Mod Carc\%), head yield (ModHead\%), headless gutted carcass yield (Mod HGCarc\%).

\begin{tabular}{|c|c|c|c|c|c|c|c|c|c|}
\hline & $\mathrm{P}$ & $\mathrm{L}$ & $\mathrm{K}$ & Carc\% & Head\% & HGCarc\% & ModCarc\% & ModHead\% & ModHGCarc\% \\
\hline $\mathrm{P}$ & $0.37 \pm 0.05$ & $0.91 \pm 0.02$ & $0.71 \pm 0.06$ & $-0.13 \pm 0.11$ & $-0.51 \pm 0.10$ & $0.15 \pm 0.11$ & $-0.26 \pm 0.12$ & $-0.68 \pm 0.07$ & $0.32 \pm 0.11$ \\
\hline $\mathrm{L}$ & 0.93 & $0.29 \pm 0.05$ & $0.34 \pm 0.10$ & $-0.04 \pm 0.11$ & $-0.39 \pm 0.09$ & $0.16 \pm 0.10$ & $-0.13 \pm 0.12$ & $-0.49 \pm 0.10$ & $0.33 \pm 0.11$ \\
\hline $\mathrm{K}$ & 0.62 & 0.29 & $0.54 \pm 0.06$ & $-0.13 \pm 0.09$ & $-0.47 \pm 0.08$ & $0.13 \pm 0.10$ & $-0.29 \pm 0.11$ & $-0.64 \pm 0.07$ & $0.19 \pm 0.12$ \\
\hline Carc\% & -0.16 & -0.08 & -0.24 & $0.49 \pm 0.06$ & $0.13 \pm 0.11$ & $0.83 \pm 0.03$ & $0.88 \pm 0.04$ & $0.26 \pm 0.11$ & $0.51 \pm 0.10$ \\
\hline Head\% & -0.61 & -0.51 & -0.5 & 0.22 & $0.47 \pm 0.06$ & $-0.45 \pm 0.09$ & $0.22 \pm 0.11$ & $0.90 \pm 0.03$ & $-0.69 \pm 0.07$ \\
\hline HGCarc\% & 0.22 & 0.23 & 0.07 & 0.8 & -0.39 & $0.55 \pm 0.06$ & $0.70 \pm 0.07$ & $-0.28 \pm 0.11$ & $0.87 \pm 0.05$ \\
\hline ModHead\% & -0.63 & -0.49 & -0.58 & 0.22 & 0.68 & -0.18 & 0.35 & $0.26 \pm 0.05$ & $-0.59 \pm 0.09$ \\
\hline ModHGCarc\% & 0.39 & 0.38 & 0.19 & 0.3 & -0.46 & 0.56 & 0.44 & -0.66 & $0.25 \pm 0.05$ \\
\hline
\end{tabular}

